\title{
confluências
}

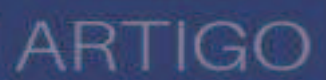

\section{CO-PRODUCIR COMÚN DESDE LA INVESTIGACIÓN SOCIAL: Reflexiones a partir de lo aprendido en una experiencia de mapeo participativo con comités de agua del municipio de Cuetzalan del Progreso, México}

Lucia Linsalata

Benemérita Universidad Autónoma de Puebla (BUAP)

Sandra Rátiva

Benemérita Universidad Autónoma de Puebla (BUAP)

Juliana Gómez

Benemérita Universidad Autónoma de Puebla (BUAP)

Kevin Hernández

Benemérita Universidad Autónoma de Puebla (BUAP)

\begin{abstract}
RESUMEN
El presente artículo sistematiza algunos aprendizajes que se han producido desde el proceso de mapeo e investigación participativa que se co-produjo entre el equipo de la línea de Entramados Comunitarios y Formas de lo Político, del posgrado de sociología de la Benemérita Universidad Autónoma de Puebla y los comités comunitarios de agua del municipio de Cuetzalan del Progreso, estado de Puebla, México. Los principales aprendizajes de este proceso giran en torno al fortalecimiento de las luchas en defensa del agua y del territorio a través de la apropiación de ciertas herramientas cartográficas, y de las posibilidades de desbordar el extractivismo académico y del capitalismo académico, que condicionan el quehacer de la investigación crítica como apoyo y respaldo del conocimiento y las prácticas de las comunidades en sus territorios.
\end{abstract}

Palabras Claves: Co-producción de lo Común. Mapeo Participativo. Investigación Social

\section{CO-PRODUÇÃO COMUM DESDE A INVESTIGACÃO SOCIAL: Reflex̃̃es a partir do aprendizado em uma experiência de mapeamento participativo com comitês de água do município de Cuetzalan del Progreso, México}

\begin{abstract}
RESUMO
$\mathrm{O}$ presente artigo sistematiza algumas aprendizagens que foram produzidas a partir do processo de mapeamento participativo e de pesquisa que foi co-produzido entre a equipe dos Entramados Comunitários y Formas de lo Político, da pós-graduação em Sociologia da Universidade Autônoma Benemérita de Puebla e os Comitês Comunitários de água do município de Cuetzalan del Progreso, estado de Puebla, México. As principais lições deste processo giram em torno do fortalecimento das lutas em defesa da água e do território através da apropriação de certas ferramentas cartográficas e as possibilidades de transpor o extrativismo acadêmico e o capitalismo acadêmico, que condicionam o trabalho de pesquisa crítica como apoio e endosso do conhecimento e práticas das comunidades em seus territórios.
\end{abstract}

Palavras-chave: Co-produção do Comum. Mapeamento Participativo. Investigação Social. 


\section{INTRODUCCIÓN}

¿Es posible, en medio de las contradicciones y de las prácticas de precarización y disciplinamiento del trabajo intelectual que el capitalismo académico nos impone cada vez con más fuerza y eficacia, poner en marcha procesos colectivos de investigación, comprometidos con las luchas sociales y respetuosos de los tiempos y las necesidades de los actores que las sostienen? ¿Es posible romper con el paradigma del investigador solitario y "extractivista" y abrirse a la construcción de procesos de co-investigación capaces de generar conocimientos "desde, con y para" las luchas con las que nos comprometemos? ¿Es posible poner en cuestión las jerarquías epistémicas y sociales que separan la academia del hacer cotidiano de los actores sociales que estudiamos? ¿Por qué tipo de límites y contradicciones atraviesan los procesos de investigación que intentan recorrer los caminos de la investigación participativa y la co-producción de conocimientos? ¿Qué potencialidades tienen? ¿Qué tipo de relaciones se producen y se cultivan en estos procesos? ¿Cómo se sostienen en el tiempo?

Estas preguntas nos han acompañado con insistencia a lo largo de los últimos tres años, durante los cuales, desde un grupo de investigación coordinado por la Prof. Lucia Linsalata e integrado por estudiantes de la maestría y la licenciatura en Sociología de la Benemérita Universidad Autónoma de Puebla, hemos intentado construir en colectivo un camino de investigación y mapeo participativo al lado de los comités comunitarios de agua del Municipio de Cuetzalan del Progreso, México. En este artículo nos proponemos sistematizar algunos de los aprendizajes que hemos cosechado a partir del camino recorrido, reflexionando colectivamente en torno a cuatro ejes de problematización.

1) En primer lugar, explicaremos de qué manera hemos buscado, en medio de múltiples dificultades, superar el problema del extractivismo académico, intentando construir, desde el diseño de la investigación, un relacionamiento que fuera constructivo y provechoso no sólo para el grupo de investigación, sino también y sobre todo para las comunidades. Explicaremos los retos que esta búsqueda nos ha puesto en frente y de qué forma nos ha llevado a repensar múltiples veces nuestro actuar en campo y a construir una relación de confianza y respeto mutuo con los comités; una relación que trasciende el ejercicio puntual de la investigación y lo tiempos impuestos por la lógica del "proyecto".

2) En segundo lugar, reflexionaremos en torno al potencial político y epistemológico del mapeo y a las posibilidades de co-producción de conocimientos, diálogo de saberes y 
fortalecimiento mutuo que hemos podido habilitar en torno a la construcción participativa de un SIG (Sistema de Información Geográfica) que tiene como finalidad organizar y sistematizar información geográfica estratégica para la gestión y defensa comunitaria del agua. Hablaremos de los usos y sentidos diferenciales que se dieron a la información producida a través de esta herramienta, a medida que tanto los comités como el equipo de investigación se fue apropiando de la misma, y de cómo, a partir de esta experiencia, se fueron tejiendo múltiples relaciones de cooperación y apoyo mutuo.

3) En tercer lugar, presentamos cómo, en la relación de co-producción de conocimiento, hemos podido construir una agenda propia de defensa del agua y cómo estamos articulando dicha agenda con nuestra disputa contra el capitalismo académico, que genera condiciones precarias y técnicas de disciplinamiento dentro de las instituciones académicas.

4) Finalmente, compartimos algunas reflexiones en torno a la forma en que hemos construido, al interior del grupo de investigación, el trabajo de co-investigación y escritura colectiva de los resultados, buscado todo el tiempo salir de la dinámica individualizante e individualizada al que nos confina el trabajo académico institucional.

No está demás aclarar que nos atrevemos a hacer este ejercicio de sistematización de lo aprendido, no con el afán de ofrecer respuestas a las preguntas que hemos puesto sobre la mesa, sino más bien con el propósito de organizar y compartir nuestra experiencia vital, en la esperanza de que, junto con otras, pueda contribuir a abrir más preguntas y a enriquecer el debate en torno al difícil camino de la investigación comprometida.

En tal sentido, exponemos nuestros aprendizajes, pues no tenemos conclusiones. Consideramos que así aportamos a la construcción de una red académica, ojalá comprometida, ojalá responsable de su hacer; pero sobretodo, esperamos contribuir desde una ética de la producción de lo común en los diversos aspectos de la vida que nos atraviesan como personas, particularmente como trabajadores y trabajadoras de las instituciones académicas.

\section{CULTIVAR LOS VÍNCULOS PARA SUPERAR EL EXTRACTIVISMO ACADÉMICO}

En la última década, sobre la sierra Norte de Puebla comenzó a pesar la amenaza del despojo extractivista del capital nacional y transnacional, que buscaba aprovechar los recursos naturales de su territorio a través de la imposición de lo que sus habitantes llaman "proyectos de muerte": megaminería, extracción de hidrocarburos mediante fracking y centrales hidroeléctricas, 
muchos de ellos relacionados entre sí. Las luchas y resistencias de la sierra Nororiental de Puebla y algunos municipios colindantes de Veracruz (Centro-oriente de México, en la sierra que desciende muy cerca del Océano Atlántico) contra esos megaproyectos encontraron un lugar de articulación en la Asamblea de los Pueblos Maseual, Totonaku y Mestizo en Defensa de la Vida y el Territorio, espacio donde coordinan y apoyan las respuestas a las amenazas, asumidas como compartidas por su alcance regional (Hernández 2019).

En el municipio de Cuetzalan, la amenaza ha intentado materializarse en varios proyectos que se han topado con la resistencia tenaz de sus habitantes. Entre los intentos se cuentan: tres concesiones mineras en el sur del municipio (en la sierra de San Manuel, zona de recarga de mantos acuíferos), la construcción de una hidroeléctrica en el río Apulco, un supermercado de la empresa Walmart, un complejo turístico y la instalación de una subestación eléctrica. Los cuetzaltecos los han enfrentado gracias a un sentido arraigado de la protección del agua y el territorio, y a la organización popular, presente en grupos de distinta naturaleza, basada en la deliberación en asambleas (Linsalata 2017) y en la organización comunitaria.

Este fuerte tejido organizativo quedó plasmado en la creación del Programa de Ordenamiento Ecológico Territorial de Cuetzalan y del Comité para el Ordenamiento Ecológico Territorial Integral de Cuetzalan (COTIC), norma y órgano concebidos de forma participativa para la planeación y protección del territorio ${ }^{1}$.

Este mismo contexto organizativo nos permitió y nos implicó plantear los términos del proyecto de investigación de forma participativa desde el principio. Las organizaciones que trabajan en el municipio no son ajenas a la vinculación con actores de diverso tipo, incluido el académico, con quienes trabajaron en la formulación del Ordenamiento y en diversas investigaciones, llevadas a cabo por programas académicos de varias universidades públicas y privadas. A sabiendas de esto, nos propusimos contribuir con las labores de defensa del territorio a fin de no realizar una investigación desligada de los esfuerzos organizativos, que condujera a un estudio dirigido únicamente al público académico y sin beneficio alguno para las comunidades estudiadas.

\footnotetext{
${ }^{1}$ La Ley General del Equilibrio Ecológico y la Protección al Ambiente de la legislación mexicana regula los programas de ordenamiento territorial en el país. En esta se prevé también la creación de un órgano encargado de su vigilancia. El COTIC fue creado por acuerdo del ayuntamiento del municipio de Cuetzalan del Progreso, después de haber pasado por un proceso exhaustivo de discusión comunitaria. Fue publicado el 3 de diciembre de 2010 en el Periódico Oficial del Estado de Puebla. Su particularidad radica en la amplitud en la conformación del comité: por lo menos 85 personas, delegadas de diversas organizaciones sociales, comunitarias y cooperativas del municipio se articulan allí para blindar al municipio de actividades económicas que afecten al territorio o a sus comunidades, principalmente indígenas del pueblo Maseual. Esta diversidad y amplitud, han hecho del COTIC un ejemplo de reapropiación social de las instancias legales y estatales que en muchos otros casos han servido para despojar a pueblos y comunidades de las decisiones que afectan a sus territorios.
} 
De esta forma buscábamos evitar caer en el extractivismo académico, el cual entendemos como una relación jerarquizada y utilitaria de producción de conocimiento en las ciencias sociales y humanas, en la que una de las partes se convierte en "los sujetos investigados", que son quienes aportan insumos, para que la otra parte, normalmente "el investigador" se encarga de procesar y sacar del contexto, y de los cuales saca beneficio en forma de mérito académico y/o económico, generando un vaciamiento y un maltrato a quienes también participaron sin ningún tipo de beneficio o retroalimentación por el conocimiento producido. El extractivismo académico no se limita a los datos, sino que incluye ideas y nociones surgidos en la experiencia comunitaria, lo que pone en riesgo la potencialidad política que pueden tener (Grosfoguel 2016).

Es importante señalar que, para nuestro caso, las organizaciones tampoco hubieran querido establecer una relación de ese tipo con nuestro equipo, porque tenían claro cuál era la tarea para la que requerían nuestra contribución: nuestra propuesta de colaboración sobre la gestión comunitaria del agua fue respaldada porque los comités de agua y las organizaciones de Cuetzalan tenían como tarea pendiente establecer mecanismos de protección del agua más específicos en el Ordenamiento. Desde nuestro primer acercamiento al campo, nos manifestaron claramente sus necesidades y de este modo se fueron definiendo las pautas de colaboración entre las partes, en las que nosotros respondimos, en la medida de nuestras capacidades, a sus necesidades e inquietudes. Surgió así el interés en la recopilación de información estratégica del territorio por el valor que tiene para su defensa. Para tal efecto, acordamos la creación de un SIG para la sistematización de datos relativos a la gestión comunitaria del agua, los cuales, a su vez, se obtuvieron mediante talleres de cartografía participativa, entrevistas, cuestionarios y recorridos de campo.

La investigación sobre la gestión comunitaria del agua nos llevó a entablar una relación estrecha con sus responsables: los comités comunitarios, los cuales se cuentan por docenas en el municipio. El trabajo conjunto fue acercándonos y constituyó la base de una relación de respeto y reconocimiento mutuo. Entramos en contacto con aquellos que pudieron y quisieron colaborar con el equipo, creando, modificando y adaptando acuerdos con cada uno. Como órganos responsables de la administración de un bien común, los comités de agua tenían que poner a consideración de la asamblea su vinculación con nosotros y las decisiones relativas a la información que manejábamos, situación que, comprensiblemente, tiene un ritmo diferente del nuestro. Entablamos una relación 
cercana especialmente con tres de ellos: el Comité Central del Agua Industrial y los comités de San Andrés Tzicuilan y Santiago Yancuitlalpan².

A lo largo del trabajo conjunto para la creación del SIG, los comités fueron externándonos sus necesidades específicas a las que procuramos responder dentro de nuestros propios límites, exponiéndoselos siempre de forma honesta. El acuerdo mismo de crear un SIG fue un enorme reto para nosotros, pues solo algunos miembros del equipo contaban con experiencia en su uso. Sin embargo, decidimos asumirlo ante el interés que los comités mostraron, pues lo consideraban una herramienta útil para su labor cotidiana, en los conflictos con usuarios, particulares y con otros comités, tal como explicaremos más adelante. No obstante, la creación del SIG no fue la única forma de colaboración: de acuerdo a la coyuntura, respondimos a los requerimientos que los comités nos compartieron como escribir, indagar información oficial, confirmar datos, generar mapas específicos para situaciones específicas, contactar entidades y organizaciones para obtener nueva información, etc., la colaboración continua, pensamos, redundó en una relación de respeto y confianza que trascendió al SIG.

Desde el planteamiento de la investigación, consideramos que sustraernos del paradigma extractivista de la ciencia significaba, asimismo, no apropiarnos de los datos que recopiláramos ni de los conocimientos que los comités tienen sobre el territorio y su gestión, sino que buscamos recuperar su experiencia en la defensa del territorio como gestores autónomos insertos en un contexto amenazado. También implica no acaparar sus luchas ni hablar en nombre de las comunidades y sus organizaciones, sino escucharlas y acompañarlas en sus ritmos y en sus caminares. De este modo, la definición de las formas de la defensa la hacen las mismas organizaciones, mientras que nosotros ponemos a su consideración las habilidades y los insumos que el equipo de investigación puede proporcionar, antes de persuadirlos de aceptarlos o imponerlos.

Somos conscientes de la posición que ocupamos, como parte de la academia, en la producción de conocimiento. Los comités tienen décadas trabajando por cuenta propia y no creemos que nuestra investigación sobre la gestión del agua deba fiscalizar el desempeño o influir en la

\footnotetext{
${ }^{2}$ El Comité Central del Agua Industrial es un comité de segundo orden, donde se articulan 16 comités comunitarios y donde se toman decisiones relativas a la infraestructura principal que comparten, al agua que consumen y a las amenazas que sobre todas estas 20 comunidades afectan; en total unas 4 mil personas de 1250 hogares están vinculadas y son beneficiadas por esta organización (Rátiva, Longoni \& Melgarejo, 2018) que reproduce prácticas de trabajo comunitario, cuidado espiritual y reciprocidad, que emanan de la cultura maseual y de un importante sentido de pertenencia al territorio. Por su parte, los comités de San Andrés Tzicuilan y Santiago Yancuitlalpan corresponden a los de dos juntas auxiliares (unidades político-administrativas menores al municipio). El de Yancuitlalpan incluye al de la localidad de Alahuacapan, que también recrean y reproducen prácticas comunitarias de trabajo mancomunado y de gestión asamblearia del agua.
} 
organización de los comités; su existencia es absolutamente autónoma y nada le debe a la academia o al estado. Al contrario, estimamos importante replantear las formas de validación del conocimiento, que forzosamente tiende a pasar por las universidades y otras instituciones estatales para ser reconocido. En este sentido, hemos buscado concertar espacios de encuentro y reflexión con otras organizaciones y luchas a nivel nacional y latinoamericano, y hemos intentado ser puente para que puedan divulgar sus esfuerzos y reconocerse con otras luchas para sumar fuerzas y conocimiento por fuera de aquel circuito instituido.

Finalmente ¿qué aprendimos en este andar? Aprendimos que rebasar el extractivismo académico implica establecer relaciones de reciprocidad y respeto con las comunidades, atender a sus esfuerzos antes que imponer nuestras necesidades, cambiando la relación investigadorinvestigado. Pudimos darle otro rumbo a la investigación en la medida en buscamos, que efectivamente tuviera sentido para las comunidades. Esto exige de nosotros flexibilidad y disposición para adaptarnos a la situación, tarea a menudo complicada dado que nosotros mismos tuvimos que emprender esfuerzos de formación en el uso de herramientas sumamente técnicas, como el SIG para cumplir con los compromisos.

\section{EL POTENCIAL EPISTEMOLÓGICO Y POLÍTICO DEL MAPEO: DE LA CO- PRODUCCIÓN DE LOS MAPAS A LOS USOS DIFERENCIALES DE LO CO- PRODUCIDO}

Tal como mencionamos, el haber habilitado un espacio de diálogo que nos permitiera diseñar la investigación con base en las necesidades, las reflexiones y los deseos que se venían cultivando al interior de los comités de agua y de las organizaciones que confluyen en el COTIC, desembocó en la decisión colectiva de elaborar un SIG que permitiera sistematizar un conjunto de informaciones estratégicas relativas a los cuerpos de agua presentes en el territorio y al manejo de los mismos por parte de las comunidades y el conjunto de los pobladores del municipio. En particular, había un fuerte interés por parte de los comités y las organizaciones integrantes del COTIC en tener un mapa claro de las decenas de redes hidráulicas comunitarias que abastecen al conjunto de las comunidades del Municipio de Cuetzalan. Las razones detrás de esta necesidad eran varias y, por cuestiones de brevedad, no profundizaremos aquí en ellas. Nos limitaremos a sintetizar solo algunos de los principales aprendizajes que hemos construido colectivamente en el proceso de co-producción de unos mapas que satisficieran esta necesidad. Haremos lo anterior con el fin de 
reflexionar sobre el potencial político y epistemológico del mapeo participativo como metodología de investigación e intervención en campo.

Como es sabido, los mapas han sido históricamente uno de los principales instrumentos que desde el poder dominante se han utilizado para ordenar los territorios, construir representaciones sobre los mismos y apropiarse de sus recursos. Las organizaciones sociales de Cuetzalan, sobre todo a nivel de sus dirigencias, tienen una idea muy clara del poder de los mapas. Durante el proceso de la elaboración participativa del Ordenamiento Territorial y de defensa del territorio de los "proyectos de muerte", han aprendido a hacer un uso profundamente estratégico de los mismos. En el transcurso de la lucha, han recurrido en más de una ocasión al contra-mapeo (al mapeo crítico y/o al mapeo participativo) como un instrumento más que les ha permitido desestructurar las estrategias empresariales y estatales de apropiación del territorio, e impulsar procesos de re-apropiación social del mismo y de control colectivo sobre los recursos comunes. En tal sentido, la necesidad de producir un mapa de la multiplicidad de las redes comunitarias de agua presentes en el territorio respondía, antes que nada, al deseo de generar un instrumento que les permitiera visibilizar, reconocer y validar la extensa presencia de las organizaciones comunitarias de agua en el territorio y, a partir de dicho reconocimiento, ordenar colectivamente los usos del agua con base en las prácticas, las necesidades y los saberes organizativos de dichas organizaciones. Ordenarlas con una doble finalidad, compartida por una variedad de organizaciones comunitarias: la de mejorar la capacidad de las comunidades de tomar decisiones conjuntas y solucionar una multiplicidad de problemas internos relativos a la gestión del agua; y la de poner límites a la apropiación privada de este bien común y a los procesos de despojo en marcha.

Lo anterior implicó que la elaboración del SIG y de los mapas que lo integrarían, fue pensada siempre, por parte de los comités de agua y de las demás organizaciones que integran el COTIC, como un instrumento interno para propiciar la toma de decisiones colectivas, y no como un instrumento meramente técnico, dirigidos a unos cuantos especialistas.

Éste ha sido el sentido político más profundo que los comités de agua depositaron desde el principio en el proyecto de elaboración del SIG. Dicho sentido emergió como anhelo colectivo desde la primera reunión que el conjunto del equipo de investigación tuvo con los comités; sin embargo, fue tomando forma y concreción, en la medida en que los comités comenzaron a relacionarse con los mapas y a apropiarse cada vez más de sus usos estratégicos.

Para quienes fuimos parte del equipo de investigación que trabajó en este proyecto, la finalidad política que los comités nos transmitieron ha representado, en cambio, una tensión fértil y 
movilizadora a lo largo de todo el proceso de investigación; una tensión que nos ha llevado a interrogarnos una y otra vez sobre nuestro papel en campo y sobre cómo teníamos que construir este proceso.

¿Cómo conciliar la elaboración de una herramienta sumamente técnica como un SIG con la toma colectiva de decisiones? ¿Cómo detonar un proceso que permitiera a los comités apropiarse realmente de esta herramienta? ¿Era realmente posible hacerlo? ¿Hasta qué punto podíamos lograrlo? Confesamos que nadie de nosotros tenía respuesta a estas preguntas al principio de este camino; las pocas respuestas que pudimos dar a estas interrogantes se fueron construyendo al andar, desde la vivencia cotidiana de las tensiones y las expectativas que el proyecto detonó, y desde el cultivo lento y paciente del diálogo y la confianza.

En medio de muchos errores, uno de los principales aciertos metodológicos que el equipo de investigación tuvo y que abonó a dirigir la construcción del SIG en la dirección política marcada por los comités, fue la decisión de privilegiar, por encima de los recorridos de georreferenciación en campo, los ejercicios colectivos de reconocimiento territorial a partir de ortofotos y fotos satelitales y de trazado colectivo de las redes hidráulicas; trazados que se elaboraron conjuntamente entre los integrantes del equipo de investigación, los fontaneros y los dirigentes de los comités de agua sobre mapas base elaborados en gabinete. Reconocemos que la decisión de mapear los sistemas comunitarios de agua a partir de procesos de reconocimiento y trazado colectivo sobre mapas bases de las tuberías y de las instalaciones de los mismos, restó mucha precisión técnica al trabajo de mapeo. Sin embargo, reconocemos también que habilitó la posibilidad de generar espacios sumamente ricos de elaboración colectiva de los mapas y de diálogos transversales entre el equipo de investigación y los comités de agua.

A lo largo de los talleres y a partir de ellos, no solo se trazaron colectivamente las redes de agua, se habilitaron momentos de reflexión sobre los problemas que algunos comités estaban viviendo en la gestión cotidiana del recurso; se generaron momentos de debate en torno a la ley nacional de agua y al régimen de concesión vigente en México; se produjo un diálogo de saberes y de intercambio de conocimientos sobre el territorio a partir de los mapas orales que los fontaneros y los dirigentes de los comités nos iban compartiendo; se construyó confianza, afecto y reconocimiento mutuo; se realizaron una multiplicidad de reuniones para evaluar entre todos los avances del proyecto y corregir una y otra vez los mapas hasta estar satisfechos con ellos; se asumieron los límites que se iban encontrando en el desarrollo del proyecto y se tomaron decisiones colectivas para marcar juntos el rumbo a seguir. 
Finalmente, la co-producción de los mapas, habilitó la co-producción de la relación de confianza: nos permitió construir un común, que trascendió la producción del SIG en sí y se transformó en la base de un vínculo de complicidad y colaboración mucho más amplio.

Un elemento esencial, a partir del cual dicho vínculo de confianza se pudo sostener y cultivar en el tiempo, fue el hecho de que el grupo de investigación respetó en todo momento dos acuerdos básicos que se tomaron desde la primera reunión. El primero de ellos fue que toda la información que se iba a producir en la elaboración del SIG era de propiedad y uso exclusivo de los comités de agua, lo cual implicaba que el equipo de investigación no podía publicarla ni pasarla a terceros, sin autorización previa de los mismos. El segundo fue que el equipo se comprometía a generar un proceso de formación y apropiación del GIS entre los comités, para que éstos pudieran llegar a ser autónomos en el uso del mismo y a no depender del equipo en futuro.

El día que tomamos ambos acuerdos no hubiéramos podido imaginar sus consecuencias futuras. En primer lugar, los comités tuvieron que hacerse cargo en más de una ocasión del hecho de ser lo propietarios de esta información y de tener que tomar decisiones colectivas en relación a los usos posibles de la misma. Lo anterior habilitó un proceso de fuerte empoderamiento en los comités que llegó a modificar las relaciones de poder, tanto al interior de los espacios de articulación política con otras organizaciones sociales, como en los espacios de negociación y diálogo con el gobierno municipal. Sería imposible dar cuenta en pocas líneas de la complejidad de los procesos deliberativos que se detonaron en torno a los usos de los mapas que se co-produjeron para el SIG. Lo único que nos interesa transmitir aquí es que uno de los logros más grandes que pudimos obtener al asumir que los comités eran los únicos propietarios de la información producida consistió en el hecho de que, como equipo, "perdimos" todo control sobre sus usos; quienes se hicieron cargo en todo momento de definir los usos estratégicos de los mapas fueron los comités y, al hacerlo, se apropiaron del SIG de forma muy peculiar. Pues, no lo hicieron de forma técnica, por lo menos no todavía, ya que el proceso de formación en esta herramienta técnica sigue en marcha y en muchos sentidos se ha quedado trunco. Sin embargo, se apropiaron de sus usos políticos y estratégicos, transformado el SIG en una herramienta de autoafirmación. En una herramienta que les permitiera decir en distintos contextos: "Existimos. Hemos sido capaces de documentar y mapear minuciosamente nuestra presencia en el territorio. Queremos y podemos hacer un 
'ordenamiento del agua' ${ }^{3}$ sin tutelajes externos y contamos con cómplices que nos pueden apoyar en ello".

Ahora bien, no hay que dejar de decir que estamos conscientes del hecho de que, sin una plena apropiación técnica de todo el proceso de elaboración de los mapas y de alimentación del SIG, los usos que los comités podrán hacer de esta herramienta serán siempre limitados. El proceso de formación y transmisión plena de esta tecnología sigue siendo un reto que no hemos podido alcanzar todavía. La brecha tecnológica que separa las comunidades de una herramienta electrónica como el SIG no es fácilmente subsanable; por otro lado, la permanente ausencia de recursos hacia este tipo de investigación y la precariedad de nuestro tiempo para realizar trabajos en campo vuelve casi imposible transformar este objetivo en realidad. Sin embargo, el acuerdo sigue de pie y, con él, el compromiso y la confianza de seguir abriendo juntos caminos que permitan fortalecer la defensa, la reapropiación y el control comunitario del agua en Cuetzalan.

¿Qué aprendimos sobre el mapeo en todo este andar? Aprendimos que el mapeo es una extraordinaria herramienta de intervención política, y co-producción de conocimientos. Es una herramienta capaz de habilitar espacios muy fértiles de diálogo e intercambio de saberes, y de detonar procesos muy importantes de representación, resignificación y politización del territorio. Sin embargo, esta capacidad no está inscrita en el mapa en sí. El contra-mapeo no tiene ningún poder, si termina en la pura elaboración de un mapa, es decir, si termina siendo una acción más de positivización y fetichización de la realidad; su potencialidad epistemológica y política se detona en la medida en que el proceso de mapeo sigue anclado a un proceso organizativo vivo capaz de resignificarlo permanentemente, de reinventarlo a cada paso y de atribuirle una multiplicidad de usos diferenciales según el contexto o la coyuntura política a enfrentar y la lucha que se está dando.

\section{LAS LUCHAS SON LUCHANDO, Y PASAN POR LA EXPERIENCIA VITAL}

En el marco de los procesos de defensa del agua y del territorio que los comités llevan a cabo, nuestro trabajo de investigación es un acercamiento posible; uno entre muchos. De forma deliberada, dialogada y discutida, el equipo de investigación ha puesto al servicio de los comités los recursos y las posibilidades que, desde la institución académica, y que desde las capacidades humanas y personales de quienes conforman el grupo, existen para fortalecer la acción y la agenda

\footnotetext{
3 Esta es la expresión que los comités de agua y las demás organizaciones que confluyen en el COTIC usan habitualmente para referirse al proyecto que cultivan desde hace años de generar de forma participativa una normatividad interna que ordene las prácticas de apropiación y uso de los cuerpos de agua presentes en el territorio, con base en las formas comunitarias de gestión y cuidado del agua.
} 
de los comités. Entendemos por agenda política, los sueños, las acciones y los planes que se priorizan y en los cuales se invierte energía vital y social para llevarlos a cabo de forma colectiva. En este sentido, es claro que la defensa del agua y del territorio es el punto en común entre la agenda de los comités y del equipo de investigación; pues es fundamental aceptar y hacer visible que el grupo que se conforma en el ámbito institucional de la universidad, también tiene una agenda, y que estos planes y acciones que provienen de este lado de la relación también se ponen en juego en la relación misma; por lo cual, el compromiso crítico con la defensa del agua como un bien común, vital y fundamental para la existencia, atraviesan entonces la relación que se gesta y que se construye.

Nuestro grupo hace parte de la línea de investigación de Entramados Comunitarios y Formas de lo Político, del posgrado de sociología del Instituto de Ciencias Sociales y Humanas de la Benemérita Universidad Autónoma de Puebla; una de las universidades públicas más grandes de México, que gracias a los fondos públicos sostiene un alto nivel entre sus posgrados y entre sus diversas investigaciones. Nuestra línea de investigación incentiva y procura el desarrollo de procesos que generen vínculos entre la universidad y las diversas experiencias comunitarias y de lucha que se despliegan en la región y en el país; esto es posible gracias a pequeños fondos que aún existen para el trabajo de campo y a la existencia de becas que se otorgan a los posgrados de alta calidad en el país, y que facilitan el trabajo de formación e investigación de los estudiantes vinculados a ellos.

A pesar de estas condiciones, que resultan favorables respecto al resto de América Latina, en un escenario global de privatización de la educación y de jerarquización de las ciencias duras y aplicadas, rentables para la innovación tecnológica que el capital exige, el ejercicio de investigación que se desplegó en Cuetzalan nos mostró las tensiones y los límites de la producción de conocimiento crítico dentro de las instituciones académicas ¿Cómo conseguir procesos de vinculación entre las universidades públicas y las organizaciones sociales y comunitarias cuando existen presiones desde el Estado para recortar gastos en las instituciones académicas? ¿Cómo generar procesos de investigación respetuosos con los tiempos, las formas y los contenidos de las comunidades cuando hay una lógica de productividad académica individualizante medida de forma numérica y anual? ¿Cómo salir de extractivismo académico cuando los salarios, las becas, los fondos y las convocatorias son los mecanismos de disciplinamiento de los y las investigadoras que hacen parte de las instituciones académicas? 
Estas preguntas, que son las contradicciones vitales que atraviesan también al equipo de investigación como personas, nos han llevado a ratificar un deseo, una voluntad y una decisión colectiva por sumarnos a la defensa del agua y del territorio como imperativo ético; imperativo que nos ha sostenido en el relacionamiento con los comités de agua, y en el fortalecimiento de un método de trabajo que co-produce, que intenciona su actuar y busca las mejores posibilidades de colaboración, tanto dentro del equipo, como con los comités, el COTIC y otras organizaciones con las que nos hemos encontrado en el camino.

En este sentido, este proceso de co-producción de conocimiento, también nos ha producido, nos ha cuestionado y nos ha permitido aclarar el lugar desde el que podemos actuar, haciendo evidentes las presiones y las amenazas que, como personas trabajadoras en las universidades e instituciones académicas, recaen sobre nosotras: la imposición de tiempos reducidos e inflexibles para "ejecutar" "proyectos" de investigación, que nada tienen que ver con los procesos con los que nos relacionamos; la instalación de la competencia entre colegas, por pequeños fondos para desarrollar trabajos en campo, que instaura la individualización y no la cooperación; la remuneración a través de estímulos de corte neoliberal que resultan de un ritmo frenético de productividad académica que no dejan tiempo para la investigación sostenida y responsable; y la presión por la "innovación” como criterio de evaluación, dejando por fuera la responsabilidad y la vinculación con la sociedad en su compleja diversidad.

Todas estas condiciones y circunstancias, que parecieran lejanas a la defensa del agua y el territorio, en realidad operan como mecanismos del capitalismo académico, que desestimula la generación de conocimiento crítico y antisistémico, y fortalece las relaciones fetichizadas al interior del campo de producción legítimo y rentable de producción de conocimiento. Si algo hemos aprendido en este ejercicio, es que los mapas son justamente una de las concreciones de conocimiento para la dominación, para la privatización y para el despojo, y que, de no disputarlos críticamente, los mapas solo serían conocimiento contra las comunidades para desdibujarles, invisibilizarles y borrarles.

Por esto mismo, parte de la agenda que se ha construido al interior del equipo y de la línea de investigación, como resultado de este proceso de co-producción con los comités, también incluye la conciencia del trabajo académico como una labor en disputa contra el capitalismo académico, lo que nos empuja a procurar e intencionar la construcción de redes colaborativas de reflexión, análisis y de intercambio de saberes; a la vez que nos ratifica sobre formas de relacionamiento con los 
comités de agua de Cuetzalan, y con otras organizaciones que también defienden el agua y sus territorios.

En este sentido, nos asumimos parte de un esfuerzo amplio y diverso por la defensa del agua y del territorio y apostamos por acciones conjuntas con otros espacios insertos en instancias académicas e institucionales, y buscamos impulsar encuentros y redes temáticas que promuevan la colaboración y el intercambio entre las organizaciones comunitarias, la academia crítica y otras instancias que potencien y amplifiquen las luchas.

Quizá en este sentido, nuestros mayores aprendizajes han sido a construir una agenda con otros y otras, y modular las energías personales y colectivas para amplificar las luchas desde el lugar en el que estamos, en este caso, desde la institución académica. Desde hace varios meses nuestra agenda ha venido cambiando también, y buscamos ahora caminar en la "Red de Luchas en defensa del agua, para la reproducción de la vida digna y la construcción de autonomía energética" donde nos encontramos con varios equipos de investigación y con algunas organizaciones sociales, que seguramente, con calma y sin prisa, seguiremos promoviendo los espacios de co-producción de conocimientos. Por ahora, por ejemplo, estamos soñando con una escuelita del agua, que sirva como espacio para compartir y reproducir el conocimiento que hemos tenido el gusto de gestar con los comités.

\section{MÁS ALLÁ DE LA ACADEMIA INDIVIDUALIZANTE}

Sabemos que los tiempos del capital buscan la totalización de la vida misma. Como lo describimos anteriormente, sabemos que la academia hace parte del proyecto capitalista que parte de la individualización y la fragmentación de los procesos colectivos. En este sentido, es posible ver cómo la institucionalización de las entidades, específicamente las educativas, y la centralización de los poderes han convergido en el mantenimiento de estructuras que reproducen la subalternización de la diferencia y las lógicas de jerarquización en los procesos de aprendizaje. La educación ha sido un instrumento funcional a la lógica de la acumulación de capital a través del lugar de legitimación del conocimiento que otorga la academia como espacio hegemónico. Conscientes de ello, este proyecto ha emprendido la búsqueda por establecer relaciones de co-producción del conocimiento, tanto con los sujetos de investigación como en la construcción misma del equipo de investigación.

El proceso de institucionalización ha convertido a la academia y los espacios de producción del conocimiento, en escenarios que deben responder a las necesidades y las lógicas del 
capital. En este sentido, se impone el tiempo del capital para la producción de investigaciones que, en nuestro caso concreto, se manifestó en la conformación de un equipo de investigación en el que, si bien todos acompañamos de manera constante y colectiva la construcción y desarrollo del proyecto, cada uno de nosotros tuvo que seguir de manera paralela en el ritmo de producción individualizada de los procesos académicos que suponen el desarrollo de las tesis de investigación, la producción de artículos y la búsqueda de las puntuaciones establecidas por la burocracia académica.

Así entonces, al tiempo que emprendimos la apuesta por la producción colectiva del conocimiento y decidimos desafiar las estructuras jerarquizadas y extractivistas propias de la academia hegemónica, cada uno de nosotros enfrentó de manera personal el manejo de sus propios recursos para subvertir la lógica del tiempo productivista y abrir un espacio de trabajo y de establecimiento de relaciones de cooperación. De esta manera, el desarrollo de este proyecto implicó una decisión que se expresó en la voluntad de cada uno de los que conformamos el equipo de investigación para abrir otros ritmos de trabajo, otras formas de establecer relaciones sociales y de enfrentar los desafíos de la co-producción de conocimientos.

El ejercicio de construir un proyecto en el que las decisiones se encuentran atravesadas por la circulación de la palabra nos permitió entender que la construcción de conocimiento tiene sentido en tanto es un proceso que genera planes de trabajo, metodologías de sistematización, de observación, pero también que destruye y recrea conceptos e ideas; la conformación de un equipo de investigación integrado en su mayoría por estudiantes de sociología nos permitió, no solamente la experimentación con herramientas de aprendizaje y retos tecnológicos como la plataforma de GIS, sino que devino en el desarrollo de estrategias de colaboración y disposición de tiempo y recursos para nuestros propios procesos de formación que se nutrieron con el desarrollo mismo del proyecto.

En el acompañamiento y desarrollo del proyecto se establecieron vínculos de solidaridad que nos permitieron resolver los vacíos que cada uno experimentaba desde sus propios lugares de formación y, al tiempo, nos permitió establecer la investigación como un proceso abierto de formación permanente que se prolonga más allá del tiempo del capital.

Para nuestro gusto, este tiempo de conversar y planear el trabajo de investigación en campo, significó liberar un tiempo para disfrutar, construir complicidad, encontrar esta agenda propia que hemos venido exponiendo y generar afecto y vínculos más allá del proyecto mismo. De esta manera, conformar y producir un colectivo de trabajo nos han posibilitado experimentar formas 
de desestructurar la individualización como lugar privilegiado de construcción del conocimiento, y esforzarnos (porque sin duda requiere de esfuerzo adicional) para pensar de forma conjunta, buscar una voz en común, escribir de forma colectiva e ir buscando también posibilidades de continuar el ejercicio más allá del proyecto, del posgrado, de la titulación o de la publicación indexada.

Así pues, a pesar de las lógicas del tiempo productivista que tuvieron un costo de manera particular para cada uno de quienes nos involucramos en el proyecto, en diferentes escalas y magnitudes, las 8 personas que en diferentes tiempos y formas participamos de este ejercicio, experimentamos convergencia de tiempos, energías e ideas en este proyecto, lo que desde la lógica que hemos intentado exponer, nos permitió romper los parámetros de separación, competencia e individualización.

Por supuesto, seguimos. Intentamos proponemos y estamos buscando la forma de que lo que hemos aprendido siga fortaleciéndonos como parte de las luchas por el agua, pero también, estamos buscando los caminos para que los vínculos que se han co-producido sigan resonando y amplificándose para que la academia y la universidad sean espacios de co-producción y no de disciplinamiento. Estamos conscientes de que es un camino largo, pero también sabemos, porque lo hemos aprendido en nuestro andar, que es una de las formas para seguir construyendo academias comprometidas, investigaciones responsables y conocimientos emancipadores. $\mathrm{Y}$ por eso, seguiremos andando.

\section{REFERENCIAS:}

GAGO, Verónica. 2017. Intelectuales, experiencia e investigación militante. Avatares de un vínculo tenso. Revista Nueva Sociedad \#268, marzo-abril.

GROSFOGUEL, Ramón. 2016. Del extractivismo económico al extractivismo epistemológico y al extractivismo ontológico: una forma destructiva de conocer, ser y estar en el mundo. Tabula Rasa, 24: 123-143. Argentina.

HERNÁNDEZ, Kevin; GOMÉZ, Juliana y LÓPEZ, David. 2018 Subir al monte. Desafíos metodológicos en el uso de Sistemas de Información Geográfica en un proyecto de investigación participativa en Cuetzalan. Revista Bajo el Volcán \# 28. ICSyH-BUAP. Puebla, México. HERNÁNDEZ, Hernández Francisco Javier. 2019. Vida, muerte y lucha en la Sierra Norte de Puebla. Una reflexión en torno a la Asamblea de los Pueblos Maseual, Totonaku y Mestizo en Defensa de la Vida y el Territorio. Tesis de maestría. Benemérita Universidad Autónoma de Puebla. México.

LINSALATA, Lucia. 2017. De la defensa del territorio maseual a la reinvención comunitariopopular de la política: crónica de una lucha. Estudios Latinoamericanos, 40: 117-136. CDMX, México. 
RÁTIVA, Sandra; LONGONI, Roberto y MELGAREJO, Manuel. 2018 La experiencia de los comités comunitarios de agua en Cuetzalan, Puebla, Revista Bajo el Volcán \# 28. ICSyH-BUAP. Puebla, México.

RISLER, Julia y ARES, Pablo. 2013. Manual de mapeo colectivo: recursos cartográficos críticos para procesos territoriales de creación colaborativa / Julia Risler y Pablo Ares. - 1a ed. Buenos Aires: Tinta Limón.

\section{AUTORES:}

\section{Lucia Linsalata}

Doctora en Estudios Latinoamericanos de la UNAM. Profesora investigadora de la Benemérita Universidad Autónoma de Puebla, Puebla, México.

E-mail: entramados.comunitarios.buap@gmail

\section{Sandra Rátiva}

Maestra en sociología de la Benemérita Universidad Autónoma de Puebla

E-mail: agarimas2@gmail.com

\section{Juliana Gómez}

Maestra en sociología de la Benemérita Universidad Autónoma de Puebla

E-mail: juliana.gomez89@gmail.com

\section{Kevin Hernández}

Licenciado en sociología de la Benemérita Universidad Autónoma de Puebla

E-mail: kehernandezmtz@gmail.com 